\title{
Binary Stellar Population Synthesis Model
}

\author{
F. Zhang ${ }^{1}$, Z. Han ${ }^{1}$ and L. Li ${ }^{1}$ \\ ${ }^{1}$ National Astronomical Observatories/Yunnan Observatory, Chinese Academy of Sciences, \\ P.O. Box 110, Kunming, Yunnan Province 650011, China \\ email: zhang_fh@hotmail.com
}

\begin{abstract}
Using Yunnan evolutionary population synthesis (EPS) models, we present integrated colours, integrated spectral energy distributions (ISEDs) and absorption-line indices defined by the Lick Observatory image dissector scanner (Lick/IDS) system, for an extensive set of instantaneous-burst binary stellar populations (BSPs) with interactions. By comparing the results for populations with and without interactions we show that the inclusion of binary interactions makes the appearance of the population substantially bluer. This effect raises the derived age and metallicity of the population.

To be used in the studies of modern spectroscopic galaxy surveys at intermediate/high spectral resolution, we also present intermediate- $(3 \AA)$ and high-resolution $(\sim 0.3 \AA)$ ISEDs and Lick/IDS absorption-line indices for BSPs. To directly compare with observations the Lick/IDS absorption indices are also presented by measuring them directly from the ISEDs.
\end{abstract}

Keywords. binaries: general, stars: evolution, galaxies: clusters: general

Binary stars play an important role in the evolution of stellar population. First, observations show that upwards of 50 per cent of the stars populating galaxies are expected to be in binary or higher-order multiple systems. Secondly, in some binary systems the evolution of the component stars are significantly different from those expected from single-star evolution. The inclusion of binaries would alter the overall appearance of the population. However, binary interactions have been neglected in some evolutionary population synthesis (EPS) studies. In our studies we assume that all stars $\left(2 \times 10^{6}\right)$ are born in binaries and born at the same time, i.e., an instantaneous binary stellar population (BSP), then model any interactions within these binaries in our Yunnan EPS models.

MODELS: A full description of Yunnan EPS model and algorithm are given in Zhang et al. $(2004,2005 \mathrm{a}, \mathrm{b})$, we refer the interested reader for them. For $Z=0.02$ standard model $\sim 11.6 \%$ of the binaries would experience Roche lobe overflow (RLOF) during the past 13 Gyr.

RESULTS: We present the integrated colours, Lick/IDS absorption-line indices and integrated spectral energy distributions (ISEDs) for BSPs at low (10-20 $\AA$ ), intermediate $(3 \AA)$ and high $(\sim 0.3 \AA)$ resolutions. The low-resolution ISEDs cover the range 91$1600,000 \AA$, intermediate- and high-resolution ISEDs in the range of $3000-7000 \AA$. The Lick/IDS absorption-line indices include 21 indices of Worthey et al. (1994), four Balmer indices defined by Worthey \& Ottaviani (1997) and 13 refined indices of Trager et al. (1998). The synthetic BSP Lick/IDS absorption indices are also measured directly from the intermediate- and high-resolution ISEDs, therefore, it becomes to be possible that compare theoretical results directly with observations. The ages of BSPs are in the range $1-15 \mathrm{Gyr}$, the metallicity are in the range $0.0001-0.03$ at low resolution and $0.004-0.03$ at intermediate and high resolutions.

In Fig. 1 we present the theoretical isochrones of $\tau=1 \mathrm{Gyr}, Z=0.02$ instantaneous BSPs with and without binary interactions. It is seen clearly that the distribution of 

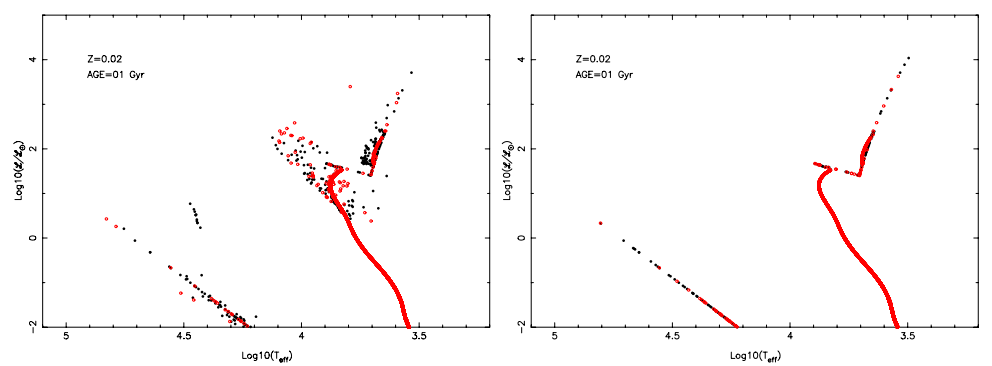

Figure 1. Theoretical isochrones for $Z=0.02$ instantaneous burst BSPs at an age of $1 \mathrm{Gyr}$ (solid, red circles for the primary, open, black for the secondary). The left and right panels represent those considering and neglecting binary interactions, respectively. For the sake of clarity only $5 \times 10^{4}$ binary systems are included in each panel.

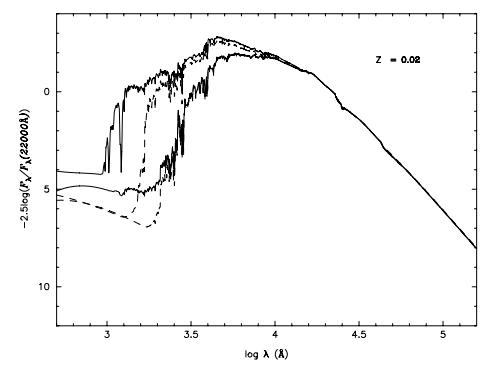

Figure 2. The ISEDs for solar-metallicity instantaneous burst BSPs with (full line) and without (dashed line) binary interactions at ages of $1 \mathrm{Gyr}$ and $13 \mathrm{Gyr}$ (upper and lower lines, respectively.

stars in Hertzsprung-Russell diagram is significantly different. These differences in the distribution of stars are responsible for differences in the appearance of EPS models.

In Fig. 2 we give a comparison of the low-resolution ISEDs for $Z=0.02$ BSPs with and without binary interactions at ages of $1 \mathrm{Gyr}$ and $13 \mathrm{Gyr}$. We see that at $\tau=1 \mathrm{Gyr}$ the ISED of BSPs with binary interactions is as much as $\sim 5$ mag, at $\tau=13$ Gyr as much as $\sim 2$ mag greater than the corresponding one without binary interactions in the farultraviolet region. In the visible and infra-red regions the BSPs with binary interactions exhibits bluer continuum than that those without binary interactions. This causes $U-B$, $B-V, V-R$ and $R-I$ colours and Lick/IDS feature indices bluer by an factor of $20 \%$, further, raises the derived age and metallicity of populations (Zhang et al. 2004, 2005a).

To satisfy the needs of modern spectroscopic galaxy survey, we also present the ISEDs and Lick/IDS absorption indices for BSPs at intermediate $(3 \AA)$ and high $(\sim 0.3 \AA)$ resolutions (Zhang et al. 2005b, 2006).

This work was in part supported by Chinese Natural Science Foundation (Grant Nos 10303006, 10673029, 10433030, 10521001), Yunnan Natural Science Foundation (Grant No. 2005A0035Q) and the Chinese Academy of Sciences (KJCX2-SW-T06).

\section{References}

Trager, S.C., Worthey, G., Faber, S.M., Burstein, D., \& Gonzalez, J.J. 1998, ApJS 116, 1

Worthey, G., Faber, S.M., González, J.J., \& Burstein, D. 1994, ApJS 94, 687

Worthey, G., \& Ottaviani, D.L. 1997, ApJS 111, 377

Zhang, F., Han, Z., Li, L., \& Hurley, J.R. 2004, A 6 A 415, 117

Zhang, F., Han, Z., Li, L., \& Hurley, J.R. 2005a, MNRAS 357, 1088

Zhang, F., Li, L., \& Han, Z. 2005b, MNRAS 364, 503

Zhang, F. \& Li, L. 2006, MNRAS 370, 1181 